\title{
Exercise-induced changes in QT interval are smaller in COPD patients and have no impact on mortality
}

\author{
To the Editor:
}

Chronic obstructive pulmonary disease (COPD) is a systemic disorder with significant pulmonary and extrapulmonary manifestations [1]. The right and left heart and the great vessels may be affected [2]. Specifically, patients with COPD (especially those with hypoxaemia) have functional alterations in cardiac autonomic modulation, manifested by tachycardia at rest, reduced baroreflex sensitivity, reduced heart rate variability and abnormal recovery of heart rate following exercise [3].

A prolonged QT interval is associated with autonomic dysfunction and delayed cardiac repolarisation [4]. In a previous study of 34 hypoxaemic COPD patients, prolongation of resting heart rate-corrected QT interval (QTc) was associated with a poorer prognosis [5], whereas in a more recent study in 234 COPD patients, QT interval, QT dispersion and heart rate-corrected QT dispersion were all independent predictors of mortality [2]. QT interval shortens during exercise due to exercise-induced autonomic responses [6], and is associated with occult myocardial ischaemia and therefore increased risk of sudden cardiac death [7]. However, no study has yet investigated the evolution of QT in COPD patients during exercise, or its potential impact on survival. Therefore, we investigated the dynamics of QT and QTc intervals during exercise in a population of stable COPD outpatients and matched healthy controls.

A retrospective analysis of prospectively collected data was undertaken. The initial patient cohort included all patients with COPD (post-bronchodilator forced expiratory volume in $1 \mathrm{~s}\left(\mathrm{FEV}_{1}\right)$ /forced vital capacity (FVC) ratio <0.7) recruited between February 1996 and June 2013 to clinical trials at our institution. We then excluded patients who were clinically unstable (i.e. acute exacerbation, hospital admission, respiratory infection or change in medication in the preceding 3 months), and those with chronic heart failure, renal impairment, known clinically relevant dysrhythmias and/or active cancer. Non-COPD controls matched for age, sex and smoking status, who were recruited between January 2011 and June 2013 as part of another study [8], constituted the initial population of controls. Only subjects with full baseline measurements of pulmonary function and gas transfer who had undergone maximal cardiopulmonary exercise testing on a cycle ergometer were included in the final study population. Survival data were available for all patients until June 2014.

ECG parameters were recorded using a 12-lead electrocardiogram pre-test and during peak exercise. QT and RR intervals were manually measured from lead II; where II was not available, lead V5 was preferred, followed by whichever other available lead obtained the best trace. For each participant the same lead was used at rest and at peak exercise. QTc was then calculated using Bazett's formula [9]. Rhythm and QRS morphology were obtained by visual interpretation of the ECG by a specialist cardiologist. The heart rate and corresponding PR intervals and QRS durations were calculated by the ECG interpretation software.

All analyses were performed using Predictive Analytics Software (version 18; SPSS Inc., Chicago, IL, USA). Normality of distribution was tested using the Shapiro-Wilk test. Data are presented as mean $\pm \mathrm{SD}$ or median (range). Group comparisons for continuous variables were conducted using either a t-test or a Mann-Whitney U-test, and the Chi-squared or Fischer's exact tests were used to compare categorical variables. Paired t-tests or a Wilcoxon rank test were used to compare the changes between rest and peak exercise variables. Cox proportional hazard analysis was used to investigate the impact of QT and QTc intervals on mortality. A value of $\mathrm{p}<0.05$ was considered significant.

@ERSpublications

COPD patients establish a flat shortening of QT interval during maximum exercise, irrespectively of medication taken http://ow.ly/XMrb308wUZt

Cite this article as: Boutou AK, Franks R, Mohan D, et al. Exercise-induced changes in QT interval are smaller in COPD patients and have no impact on mortality. Eur Respir J 2017; 49: 1602394 [https://doi. org/10.1183/13993003.02394-2016]. 
The study population consisted of 143 patients (65.7\% male; mean \pm sD age $62.4 \pm 7.7$ years; $\mathrm{FEV}_{1} 32.3 \pm 14 \%$ ) and 18 healthy controls, matched for age $(65.7 \pm 8.5$ years, $\mathrm{p}=0.093)$ and gender $(66.7 \%$ male; $\mathrm{p}=0.937)$. Several differences were noted between patients and controls regarding rest and peak exercise ECG variables. Patients established a shorter resting QT $(333 \pm 34 \mathrm{~ms}$ versus $361 \pm 39 \mathrm{~ms} ; \mathrm{p}=0.002)$ and longer exercise QT $(299 \pm 32 \mathrm{~ms}$ versus $270 \pm 27 \mathrm{~ms} ; \mathrm{p}<0.001)$ than controls. However, these differences were reduced when resting QTc and exercise QTc were compared between the groups, because patients had a significantly higher resting heart rate $\left(90.8 \pm 16.6\right.$ beats $\cdot \mathrm{min}^{-1}$ versus $76.7 \pm 22.4$ beats $\left.\cdot \mathrm{min}^{-1} ; \mathrm{p}=0.001\right)$ and lower exercise heart rate $\left(115.3 \pm 18.2\right.$ beats $\cdot \mathrm{min}^{-1}$ versus $147.7 \pm 19.9$ beats $\left.\cdot \mathrm{min}^{-1} ; \mathrm{p}<0.001\right)$ than controls. For the other ECG variables, resting PR (154 (83-283) ms versus $165(142-221) \mathrm{ms} ; \mathrm{p}=0.001)$ and resting RR intervals $(682.5 \pm 140.2 \mathrm{~ms}$ versus $838.9 \pm 167.4 \mathrm{~ms} ; \mathrm{p}<0.001)$ were significantly shorter in the patient group, whereas exercise $\mathrm{PR}(154$ (83-283) ms versus $129(80-192) \mathrm{ms} ; \mathrm{p}=0.002)$ and exercise $\mathrm{RR}$ intervals $(532.3 \pm 90.9 \mathrm{~ms}$ versus 410.0 $\pm 58.3 \mathrm{~ms} ; \mathrm{p}<0.001$ ) were significantly longer in the patient group than in the control group.

The dynamics of QT, QTc and other ECG parameters in both groups are presented in table 1. From rest to peak exercise, QT interval in the control group shortened $(361 \pm 39 \mathrm{~ms}$ versus $270 \pm 27 \mathrm{~ms}, \mathrm{p}<0.001)$, and QTc increased $(396 \pm 24 \mathrm{~ms}$ versus $422 \pm 32 \mathrm{~ms}, \mathrm{p}<0.001)$; in COPD patients, the QTc interval tended to increase, and QT interval shortened $(333 \pm 34 \mathrm{~ms}$ versus $299 \pm 32 \mathrm{~ms} ; \mathrm{p}<0.001)$; however, the absolute change of QT from rest to peak exercise in the patient group was about $35 \%$ of that seen in controls. Considering other ECG variables, RR interval shortened in both groups, but again the absolute change in the control group was almost four times that in the patient group. QRS remained unchanged in both groups, and PR shortened only in controls. Median survival for the patient population was 101.4 (61.5141.3) months, but neither QT nor QTc at peak exercise were associated with mortality.

The patient population was further divided into three groups, based on previous medication history: group 1 (those who had received medication known to prolong QT interval, such as quinolones, macrolides and antipsychotic drugs within the previous 6 months; $n=36$ ); group 2 (those who had not received such drugs within the previous 6 months; $\mathrm{n}=51$ ); and Group 3 (unknown medication status; $\mathrm{n}=56$ ). The KruskalWallis $\mathrm{H}$ test indicated that neither $\Delta \mathrm{QT}$ nor $\Delta \mathrm{QTc}$ differed significantly between the groups $(\Delta \mathrm{QT}$ : group 1, 20 (-20-100) ms; group 2, $40(0-100) \mathrm{ms}$; group 3, $40(-120-100) \mathrm{ms}, \mathrm{p}=0.226$; $\Delta \mathrm{QTc}$ : group 1, $1(-57-71) \mathrm{ms}$; group 2, $4(-39-66) \mathrm{ms}$; group 3, 1 (-62-69) ms, p=0.970).

We show, for the first time, that individuals with severe COPD have different ECG responses to maximum exercise compared to matched controls. In control participants, the QT interval shortened and QTc increased from rest to peak exercise; however, the response in COPD patients was flat and almost one third of that seen among controls. The reason for this difference was not explained in the present study, and the effect does not seem to have a significant impact on COPD survival. Previous data indicated that patients with COPD have altered resting cardiac repolarisation with prolonged QTc interval and QT dispersion compared to healthy controls [10]; however, that study was conducted with patients whose disease was less severe than in the present study, and participants with known arrhythmias and other common disorders associated with QT prolongation were not excluded.

The findings of the present study may, to some extent, be limited by the retrospective design and relatively small number of controls. However, previous studies included fewer patients to identify disorders of cardiac repolarisation in COPD patients at rest $[5,10]$. Moreover, the present study results are strengthened by the fact that data were collected prospectively, all cardiopulmonary exercise testing was conducted in the same centre and using the same equipment, and all ECGs were evaluated by the same cardiologist.

In conclusion, this is the first study to establish that although cardiovascular dynamics in patients with severe COPD differ from those in control participants, COPD patients do not demonstrate QT prolongation

TABLE 1 Dynamics of ECG parameters in patient and control groups

\begin{tabular}{lcccr} 
ECG variables & Patients & p-value & Controls & p-value \\
\hline Subjects n & 143 & & 18 & \\
Exercise QT-resting QT ms & $-34 \pm 29$ & $<0.001$ & $-91 \pm 34$ & $<0.001$ \\
Exercise QTc-resting QTc ms & $5 \pm 34$ & 0.053 & $26 \pm 25$ & $<0.001$ \\
Exercise QRS-resting QRS ms & $1.7 \pm 13.1$ & 0.128 & $0.2 \pm 6.2$ & 0.881 \\
Exercise PR-resting PR ms & $1(-63-100)$ & 0.057 & $-31(-104-0)$ & $<0.001$ \\
Exercise RR-resting RR ms & $-150.5 \pm 100.6$ & $<0.001$ & $-428.9 \pm 168.9$ & $<0.001$
\end{tabular}

Data are presented as mean \pm SD or median (range). ECG: electrocardiogram; QTc: heart rate-corrected QT interval. 
during maximum exercise, irrespectively of the medication taken. These data are reassuring for those supervising exercise training programmes and consistent with the accepted safety of such programmes.

Afroditi K. Boutou ${ }^{1}$, Russell Franks ${ }^{1}$, Divya Mohan ${ }^{1}$, Lilian Mantziari ${ }^{2}$, Tom Wong ${ }^{2}$, Nicholas S. Hopkinson ${ }^{1}$ and Michael I. Polkey

${ }^{1}$ NIHR Respiratory Biomedical Research Unit at Royal Brompton and Harefield, NHS Foundation Trust, London, UK. ${ }^{2}$ NIHR Cardiovascular Biomedical Research Unit at Royal Brompton and Harefield, NHS Foundation Trust, London, UK.

Correspondence: Afroditi K. Boutou, Intensive Care Unit, “G. Gennimata” Hospital, 54635, Thessaloniki, Greece.

E-mail: afboutou@yahoo.com

Received: Dec 062016 | Accepted after revision: Dec 172016

Support statement: A.K. Boutou was a recipient of an ERS Short Term Research Fellowship when the study was conducted.

Conflict of interest: Disclosures can be found alongside this article at erj.ersjournals.com

\section{References}

1 Stockley RA, Mannino D, Barnes PJ. Burden and pathogenesis of chronic obstructive pulmonary disease. Proc Am Thorac Soc 2009; 6: 524-526.

2 Zulli R, Donati P, Nicosia F, et al. Increased QT dispersion: a negative prognostic finding in chronic obstructive pulmonary disease. Intern Emerg Med 2006; 1: 279-286.

3 Van Gestel AJ, Steier J. Autonomic dysfunction in patients with chronic obstructive pulmonary disease (COPD). $J$ Thorac Dis 2010; 2: 215-222.

4 Khandoker AH, Imam MH, Couderc JP, et al. QT variability index changes with severity of cardiovascular autonomic neuropathy. IEEE Trans Inf Technol Biomed 2012; 16: 900-906.

5 Stewart AG, Waterhouse JC, Howard P. The QTc interval, autonomic neuropathy and mortality in hypoxaemic COPD. Respir Med 1995; 89: 79-84.

6 Lecocq B, Lecocq V, Jaillon P. Physiologic relation between cardiac cycle and QT duration in healthy volunteers. Am J Cardiol 1989; 64: 481-486.

7 Yi G, Crook R, Guo XH, et al. Exercise-induced changes in the QT interval duration and dispersion in patients with sudden cardiac death after myocardial infarction. Int J Cardiol 1998; 63: 271-279.

8 Mohan D, Lewis A, Patel MS, et al. Using laser capture microdissection to study fiber specific signalling in locomotor muscle in COPD: a pilot study. Muscle Nerve 2016 [accepted for publication. Manuscript ID: MUS-16-0241.R2].

9 Bazett HC. An analysis of the time-relations of electrocardiograms. Heart 1920; 7: 353-370.

10 Noriane A, Sievi NA, Clarenbach CF, et al. High prevalence of altered cardiac repolarization in patients with COPD. BMC Pulm Med 2014; 14: 55. 